\title{
Topological Sound and Flocking on Curved Surfaces
}

\author{
Suraj Shankar, ${ }^{1,2}$ Mark J. Bowick, ${ }^{1,2}$ and M. Cristina Marchetti ${ }^{1,2}$ \\ ${ }^{1}$ Physics Department and Syracuse Soft Matter Program, Syracuse University, \\ Syracuse, New York 13244, USA \\ ${ }^{2}$ Kavli Institute for Theoretical Physics, University of California, Santa Barbara, California 93106, USA \\ (Received 27 April 2017; revised manuscript received 30 June 2017; published 7 September 2017)
}

Active systems on curved geometries are ubiquitous in the living world. In the presence of curvature, orientationally ordered polar flocks are forced to be inhomogeneous, often requiring the presence of topological defects even in the steady state because of the constraints imposed by the topology of the underlying surface. In the presence of spontaneous flow, the system additionally supports long-wavelength propagating sound modes that get gapped by the curvature of the underlying substrate. We analytically compute the steady-state profile of an active polar flock on a two-sphere and a catenoid, and show that curvature and active flow together result in symmetry-protected topological modes that get localized to special geodesics on the surface (the equator or the neck, respectively). These modes are the analogue of edge states in electronic quantum Hall systems and provide unidirectional channels for information transport in the flock, robust against disorder and backscattering.

DOI: 10.1103/PhysRevX.7.031039

Subject Areas: Metamaterials, Soft Matter

\section{INTRODUCTION}

Flocking, defined as the self-organized ordered motion of collections of self-propelled units [1,2], has been the flagship of active matter for some time now [3,4]. Active entities dissipate energy to perform work and generate motion, leading to sustained and local breaking of detailed balance. Examples abound in the living world, ranging from bird flocks [5] to bacterial suspensions [6] and migrating cells [7], and include synthetic analogues, such as reconstituted cytoskeletal extracts [8-10], vibrated granular media [11,12], and self-propelled colloids [13]. Active matter as a field aims to provide broad organizational principles applicable to a wide class of these nonequilibrium systems over many scales.

Collective cellular motion on curved surfaces is ubiquitous in developmental processes, such as morphogenesis and embryonic development [14-16], or when cells migrate in the gut $[17,18]$ or on the surface of the growing cornea [19], and it also affects cell division [20]. Recent in vitro work has demonstrated a direct effect of substrate curvature on cytoskeletal alignment and cell motility in epithelial cells [21]. Understanding the behavior of active matter on curved surfaces or confined by curved boundaries is therefore timely. There has been growing recent interest in understanding this at a fundamental level, with the focus divided between the effect of curved confining walls on

Published by the American Physical Society under the terms of the Creative Commons Attribution 4.0 International license. Further distribution of this work must maintain attribution to the author(s) and the published article's title, journal citation, and DOI. so-called scalar (nonaligning) active matter [22-26] and on aligning active matter systems, of either nematic [9,10,27] or polar [28,29] symmetry. Even at the level of noninteracting self-propelled particles, the curvature of confining walls can yield surprising features, such as inhomogeneous density and pressure profiles [22,25] and the breakdown of an equilibrium interpretation [23,24]. In the presence of aligning interactions that promote orientational order, curvature has an even more profound effect since it frustrates such order, often requiring topological defects [30] that, in active systems, become dynamical and are capable of driving spatiotemporal patterns and complex motion [10]. With flexible walls or membranes present, activity can lead to spontaneous motion and rectification [31,32].

A generic property of the ordered state of polar active matter is spontaneous flow and thus the breaking of timereversal symmetry [4]. It is known that carefully engineered lattice structures with flows induced either spontaneously by activity [33] or through an external drive [34] can host exotic unidirectional sound modes that are localized at the edges of the sample and are topologically protected. The presence of topologically protected edge states in classical phononic [35,36] and photonic [37] systems has led to extensive exploration of topological metamaterials, with properties akin to electronic topological insulators and quantum Hall states [38]. Here, we show that a polar active fluid on a curved substrate supports similar topologically protected modes, even in the absence of any underlying periodicity or lattice structure. This should be contrasted with many of the systems considered previously, which required a carefully designed metamaterial structured on an artificial lattice. The phenomenon reported here 
is akin to the one recently found in geophysical flows of oceans or the Earth's atmosphere, where equatorially trapped Kelvin waves were reinterpreted as topologically protected modes [39]. In that case, the flow is imposed externally by the Earth's rotation. In our active fluid, in contrast, flow occurs with no external drive, resulting in spontaneous topologically protected modes.

The presence of these topological modes relies on three important ingredients:

(i) the spontaneous polar order and associated flow that breaks time-reversal symmetry;

(ii) the fact that in polar active fluids the order parameter also plays the role of a flow velocity, resulting in distinctly nonequilibrium self-advection not present in equilibrium polar fluids [4];

(iii) the nonzero Gaussian curvature of the underlying substrate.

We emphasize that the long-wavelength, topologically protected modes discussed here are generic, in the sense that they occur for active polar flow on any curved surface of nonvanishing Gaussian curvature. Recent work has considered active polar patterns on a cylinder [40]. In this case, the Gaussian curvature vanishes, and there are consequently no topologically protected sound modes. In the following, we demonstrate the phenomenon explicitly for flocking on the sphere, which has constant positive curvature, and on the catenoid, which has negative, spatially varying curvature.

In Sec. III, we analyze the continuum Toner-Tu model of flocking on a sphere and analytically compute the steadystate configuration of the polar-ordered flock. Because of the curvature, the ordered state is forced to be inhomogeneous, in general. On a sphere, polar order additionally requires topological defects or vortices (the "hairy-ball theorem" [41]) in order to satisfy the constraints imposed by the global topology of the surface. With a wellmotivated ansatz, we show that the covariant hydrodynamic model is capable of predicting generic inhomogeneous steady ordered phases that accommodate the curvature and topology of the underlying surface in a natural fashion. The steady flocking state on the sphere corresponds to the rotating band seen in recent particle simulations by Sknepnek and Henkes [29] and is a novel find in itself as it is peculiar to the active system (a passive polar liquid crystal on a sphere would have a very different equilibrium profile).

Having obtained a steady ordered state, in Sec. III B, we examine its excitations. Even though our system is overdamped because of the presence of a substrate, the ordered polar flock supports long-wavelength propagating sound modes [42]. The presence of curvature introduces an additional length scale in the problem and gaps the sound spectrum at long wavelengths. In other words the propagation rate of long-wavelength density fluctuations is finite. This is a distinct property of active polar fluids, and it arises because the polarization field plays the dual role of the order parameter and flow velocity and is therefore subject to the same lensing effect that forces flow to move along geodesics on curved surfaces [29,39]. With a spectral gap opened, in Sec. IV, we show that a polar-ordered flock on a curved surface supports topologically protected sound modes that are localized to special geodesic curves on the surface (at which the gap in the spectrum closes). In Sec. V, we compute the steady state of an ordered polar flock on a catenoid and show that topological modes are also present on this negative-curvature surface. In contrast to the setup of Souslov et al. [33], where the lattice structure was instrumental (along with the active flow) in generating a gapped spectrum at intermediate frequencies, here the curvature itself generates it, though now at long wavelengths, implying that the result is quite general.

\section{TONER-TU EQUATIONS ON A CURVED SURFACE}

We consider an active polar fluid on a two-dimensional (2D) surface. To make generic predictions independent of specific microscopic realizations, we work in the continuum limit and use the well-tested hydrodynamic description of a fluid of overdamped self-propelled particles provided by the Toner-Tu equations $[2,43,44]$, appropriately modified to account for the curvature of the sphere [28]. Mass conservation implies a continuity equation for the density field, $\rho$,

$$
\partial_{t} \rho+\nabla_{\mu} p^{\mu}=0
$$

with $\mu=\theta, \varphi$ and $\mathbf{p}=\rho \mathbf{u}$ the polarization density of the active fluid. Because of the presence of a substrate, momentum is not conserved, and the particle's velocity is assumed to be aligned with its direction of self-propulsion, leading to the identification of $\mathbf{u}$ with the flow velocity of the active fluid. Note that on a curved surface, parallel transport of vectors requires the use of covariant derivatives [45],

$$
\nabla_{\mu} p^{\nu}=\partial_{\mu} p^{\nu}+\Gamma_{\alpha \mu}^{\nu} p^{\alpha},
$$

where $\Gamma_{\alpha \mu}^{\nu}$ are the appropriate Christoffel symbols. The equation for the polarization density is given by

$$
\begin{aligned}
\partial_{t} p^{\mu}+\lambda p^{\nu} \nabla_{\nu} p^{\mu}= & {\left[a\left(\rho-\rho_{c}\right)-b g_{\alpha \beta} p^{\alpha} p^{\beta}\right] p^{\mu} } \\
& +\nu\left(\Delta p^{\mu}+K_{G} p^{\mu}\right)+\nu^{\prime} \nabla^{\mu} \nabla_{\nu} p^{\nu}-v_{1} \nabla^{\mu} \rho .
\end{aligned}
$$

Note that, here, u plays the dual role of an order parameter field (polarization) and velocity, as discussed in Ref. [4]. The transport coefficients $\nu$ and $\nu^{\prime}$ are the shear and bulk viscosities (or anisotropic elastic constants), respectively, $a, b>0$ are coefficients setting the magnitude of the mean-field polarized state for $\rho>\rho_{c}$ (the critical 
density for the flocking transition), $\lambda$ is a kinematic convective parameter, and $v_{1}>0$ is a compressional modulus. The last term on the right-hand side of Eq. (3) is the first term in a density expansion of the gradient of the swim pressure $[46,47]$ that describes the flux of propulsive forces across a unit plane of material. There are other nonlinear terms in the original Toner-Tu equations, but we only retain the most important ones here. In particular, we keep the convective nonlinearity $\lambda \mathbf{p} \cdot \nabla \mathbf{p}$, which is responsible for long-ranged order in 2D $[43,48]$, and the leading density dependence in the symmetry breaking $(a \rho \mathbf{p})$ and pressurelike terms $\left(v_{1} \nabla \rho\right)$ that lead to dynamical selfregulation [49], phase-separation [50-52], and longwavelength instabilities of the ordered phase $[53,54]$. The absence of Galilean invariance means that $\lambda \neq 1 / \rho$. Additional nonlinear advective terms $\sim \lambda_{2} \mathbf{p} \nabla \cdot \mathbf{p}, \lambda_{3} \nabla|\mathbf{p}|^{2}$ are also present, in general, but do not qualitatively change our results below (see Appendix A for an analysis with $\lambda_{2}, \lambda_{3} \neq 0$ ).

Curvature enters Eq. (3) in two crucial places (apart from the covariant derivatives): (i) The cubic term setting the magnitude of the polarization explicitly involves the metric tensor $\mathfrak{g}\left(|\mathbf{p}|^{2}=g_{\alpha \beta} p^{\alpha} p^{\beta}\right)$ and (ii) the Gaussian curvature $K_{G}$ explicitly appears in the viscous term because the strain rate tensor is a symmetrized derivative of the velocity and the covariant derivatives do not commute. The presence of $K_{G} \neq 0$ is a direct dynamical consequence of the PoincaréHopf theorem [41] from which it follows that topological defects or vortices are required to accommodate vector order on a curved closed surface like the sphere. A covariant hydrodynamic treatment of active fluids on a curved surface has also been developed by Fily et al. [28]. These authors derived the continuum equations by coarse graining a microscopic model of self-propelled particles, which allowed an explicit computation of the transport coefficients in terms of microscopic parameters. The form of the continuum equations obtained in Ref. [28] is identical to those used here, the only distinction being that $\mathcal{O}\left(\nabla^{2}\right)$ terms are neglected in that work, including the explicit $K_{G}$ term. In the following, we similarly neglect $\nabla^{2}$ terms.

\section{POLAR FLOCK ON A SPHERE}

As an example of a curved surface with constant positive curvature, we consider an active polar flock on the surface of a sphere of radius $R$. In local spherical polar coordinates $\{\theta, \varphi\}$, the canonical metric and curvature on $S^{2}$ are

$\mathfrak{g}=R^{2}\left(\mathrm{~d} \theta \otimes \mathrm{d} \theta+\sin ^{2} \theta \mathrm{d} \varphi \otimes \mathrm{d} \varphi\right), \quad K_{G}=\frac{1}{R^{2}}$.

The only nonvanishing Christoffel symbols are

$$
\Gamma_{\varphi \varphi}^{\theta}=-\sin \theta \cos \theta \quad \text { and } \quad \Gamma_{\theta \varphi}^{\varphi}=\cot \theta .
$$

\section{A. Steady state of a polar flock on the sphere}

At low mean density $\left(\rho_{0}<\rho_{c}\right)$, the isotropic phase with constant density and $\mathbf{p}=\mathbf{0}$ is stable. For $\rho_{0}>\rho_{c}$, where the mean-field solution in flat space is a state of constant density and finite, but uniform polarization, on the sphere one obtains polar, spatially varying states. Since the particle number is conserved, there can be no sinks or sources of flow. The simplest configuration allowed by the required conservation of topological defect charge that must sum up to the Euler characteristic $\chi=2$ of the sphere is then a circulating band wrapping around an equator, with two vortices of charge +1 at the two opposing poles. This yields a density band with polarization in the azimuthal direction and both density and polarization vanishing at the poles, consistent with the band state reported recently in simulations of polar particles on the sphere [29].

An explicit solution can be found analytically by assuming azimuthal symmetry, with $\rho=\rho_{s s}(\theta), p^{\theta}=0$, and $p^{\varphi}=p_{s s}^{\varphi}(\theta)$. The continuity equation is then satisfied identically. To simplify the polarization equation, we neglect the viscous terms as they are higher order in gradients (suppressed by $1 / R^{2}$ ) compared to the other terms arising from self-propulsion. In the microscopic realization of self-propelled polar particles with repulsive short-range forces and aligning interaction studied in Ref. [29], this approximation corresponds to the regime where interparticle repulsion (contributing to pressure) and active self-propulsion dominate over viscous and elastic stresses. We then neglect the Laplacian terms entirely by setting $\nu=0$ (the bulk viscosity $\nu^{\prime}$ drops out with our assumption of azimuthal symmetry). This leaves us with

$$
\begin{gathered}
\lambda \sin \theta \cos \theta\left(p_{s s}^{\varphi}\right)^{2}=\frac{v_{1}}{R^{2}} \partial_{\theta} \rho_{s s}, \\
p_{s s}^{\varphi}\left[a\left(\rho_{s s}-\rho_{c}\right)-b R^{2} \sin ^{2} \theta\left(p_{s s}^{\varphi}\right)^{2}\right]=0 .
\end{gathered}
$$

Writing $X(\theta)=\rho_{s s}(\theta)-\rho_{c}$, and seeking a solution with $p_{s s}^{\varphi} \neq 0$, we can eliminate $p_{s s}^{\varphi}$ from the two equations to obtain

$$
\frac{\mathrm{d} X}{\mathrm{~d} \theta}=\left(\frac{a \lambda}{b v_{1}}\right) \cot \theta X \Rightarrow X(\theta)=X(\pi / 2)(\sin \theta)^{\eta},
$$

where

$$
\eta=\frac{\lambda a}{b v_{1}}
$$

is a dimensionless parameter that controls the shape of the solution, with $\eta>0$ for the density profile to be a physical solution (note that $\sin \theta>0$ over the entire range $\theta \in[0, \pi])$. By symmetry, the density will be maximum at $\theta=\pi / 2$ (the equator). Letting $\rho_{s s}(\pi / 2)=\rho_{\max }$, we find 


$$
\rho_{s s}(\theta)=\rho_{c}+\left(\rho_{\max }-\rho_{c}\right) \sin ^{\eta} \theta .
$$

We stress that the dependence on $R$ has dropped out from Eq. (10), which therefore represents a universal density profile for an ordered flock on any size sphere. Finally, we express $\rho_{\max }$ in terms of the average density $\rho_{0} \equiv\left\langle\rho_{s s}\right\rangle$ by requiring

$$
\rho_{0}=\frac{R^{2}}{4 \pi R^{2}} \int_{0}^{2 \pi} \mathrm{d} \varphi \int_{0}^{\pi} \mathrm{d} \theta \sin \theta \rho_{s s}(\theta)
$$

to obtain the final expression for the density profile as

$$
\rho_{s s}(\theta)=\rho_{c}+\left(\rho_{0}-\rho_{c}\right) A_{\eta} \sin ^{\eta} \theta,
$$

with $A_{\eta}=2 \Gamma((3+\eta) / 2) /[\sqrt{\pi} \Gamma(1+\eta / 2)]$. In order for this density profile to exist, we additionally require that $\left|\mathbf{p}_{s s}\right|^{2}>0$ and obtain

$$
\left|\mathbf{p}_{s s}\right|^{2}=\frac{a}{b}\left(\rho_{0}-\rho_{c}\right) A_{\eta} \sin ^{\eta} \theta .
$$

As expected, an ordered flock only exists for $\rho_{0}>\rho_{c}$, and the magnitude of the steady-state polarization and the density have the same inhomogeneous profile as shown in Fig. 1(a), with the direction of polarization chosen spontaneously.

This band solution is unrelated to the traveling bands found in flat space [51-53], which occur close to the meanfield transition and are absent deep in the ordered phase. The inhomogeneous solution obtained is simply the ordered flocking state on a sphere. The spatially inhomogeneous profile arises from the interplay of mass fluxes $\left(\sim v_{1} \nabla \rho\right)$ and convective fluxes $(\sim \lambda \mathbf{p} \cdot \nabla \mathbf{p})$ that cannot be driven to zero on a curved surface. Hence, the spatial inhomogeneity is made inevitable by curvature. The present solution is expected to break down within a region of angular width $\theta_{\mathrm{m}} \sim \exp \left(-a \rho_{c} R^{2} / \nu\right)$ (hence, it is exponentially small on a large sphere) around the poles of the sphere, at the core of the vortices, as the elastic stresses will become important at short scales. Therefore, the profile obtained is a robust and universal prediction of the continuum theory, similar to the rotating band seen in particle simulations of Ref. [29].

For an equilibrium polar or ferroelectric liquid crystal (say, a compressible lyotropic smectic-C film [55]), where the polarization is strictly an order parameter field and does not play the role of a velocity, the important convective nonlinearity in Eq. (3) is absent $\left(\lambda=0\right.$, though $\lambda_{2}$ and $\lambda_{3}$ can be present [56]), and the band solution we have here is absent ( $\eta=0$, see Fig. 2). In this case, even in the ordered phase, the density remains homogeneous, and on a large enough sphere, we have nearly uniform polar order everywhere $\left(\left|\mathbf{p}_{s s}\right| \simeq\right.$ const $)$, but for two isolated defects at the

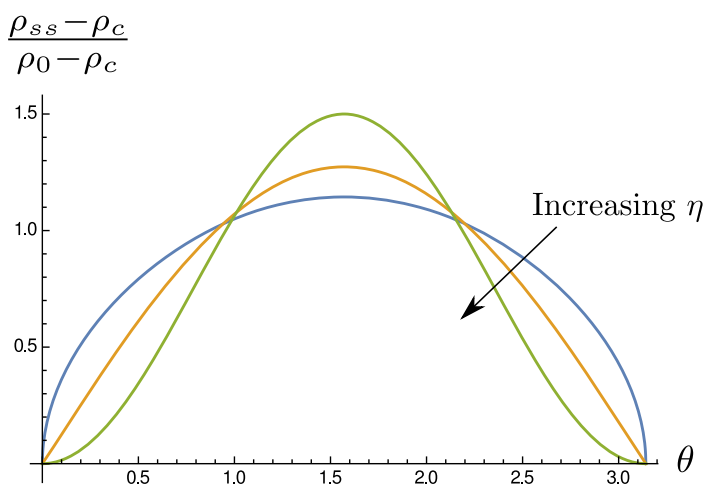

(a)

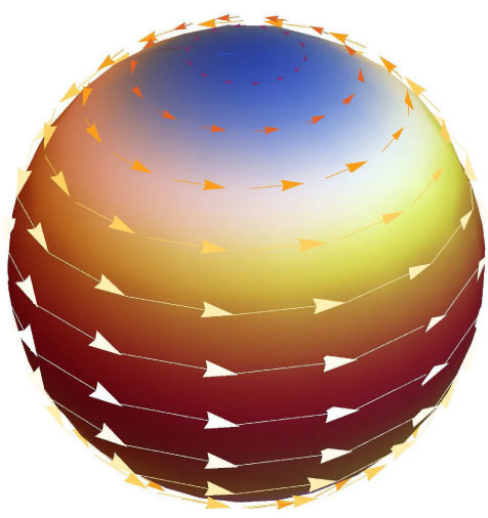

(b)

FIG. 1. (a) The normalized density profile of a polar flock on a sphere given in Eq. (12), for $\eta=0.5$ (blue), 1 (orange), and 2 (green). (b) The density and polarization profiles for $\eta=2$, now shown on the sphere. The color describes the density from the maximum (red) at the center of the polar band to $\rho_{c}$ (blue) at the poles of the sphere. The polarization also vanishes at the poles.

poles, whose core size $\xi \sim \sqrt{\nu / a\left(\rho_{0}-\rho_{c}\right)}$ is a microscopic length scale deep into the ordered state.

\section{B. Linearizing about the steady state}

Well below the mean-field transition $\left(\rho_{0}<\rho_{c}\right)$, the isotropic disordered state $\left(\rho_{s s}=\rho_{0}\right.$ and $\left.\mathbf{p}_{s s}=\mathbf{0}\right)$ is linearly stable at long wavelengths, with fluctuations in the polarization relaxing quickly and density perturbations relaxing diffusively at long times, just as in the plane [57]. The curvature does not affect the disordered phase. It is only in the ordered state that we find novel excitations with nontrivial topological properties.

Here, we consider the linear dynamics of small-amplitude perturbations about the steady ordered flocking state, letting $\rho=\rho_{s s}(\theta)+\delta \rho$ and $\mathbf{p}=\mathbf{p}_{s s}(\theta)+\delta \mathbf{p}$. We focus on the long-wavelength propagating sound modes that are present even in the plane for an ordered flock [42], and we continue to neglect all the viscous and elastic couplings. These are higher order in gradients and only give rise to damping of the sound modes. As the base state we are linearizing about is inhomogeneous, we additionally 


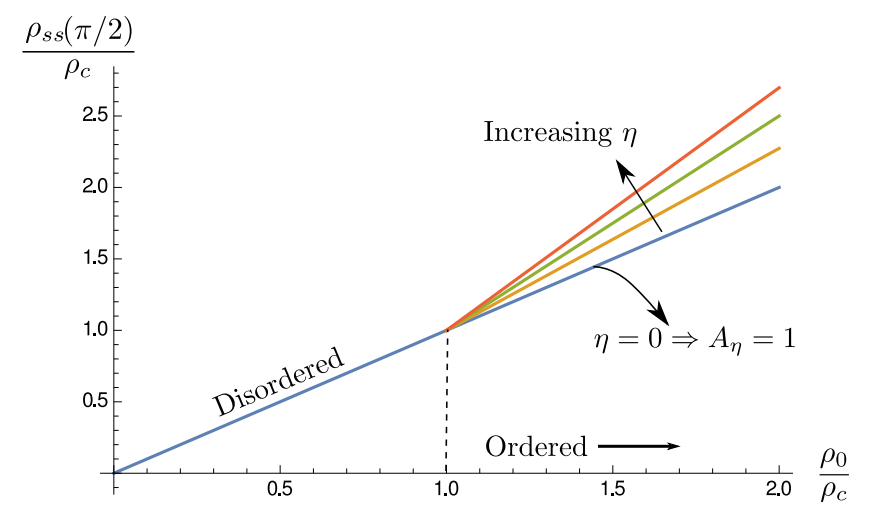

FIG. 2. The peak (maximum) density at the equator on a sphere, as we vary the mean density $\rho_{0}$. For $\rho_{0}<\rho_{c}$, we are in the disordered phase with $\rho_{s s}(\pi / 2)=\rho_{0}$. For $\rho_{0}>\rho_{c}$, we have a polar band with the density profile given in Fig. 1 . The density reaches its maximum at the center of the band and grows with $\rho_{0}$, with a slope $A_{\eta}>1$. When the convective parameter $\lambda \rightarrow 0$, $\eta \rightarrow 0$, resulting in $A_{\eta} \rightarrow 1$, and we go back to the homogeneous profile as in the flat plane.

confine ourselves to a tangent plane linearization about a fixed latitude away from the poles (a preferred local coordinate system is picked out spontaneously by the polar order, allowing for an unambiguous notion of latitude). Setting $\theta=\theta_{0}+y$ for a given latitude $\theta_{0}$, with $\theta_{0}<\pi / 2$ corresponding to the northern hemisphere and $\theta_{0}>\pi / 2$ to the southern hemisphere, relabeling $\varphi$ as $x$, and letting $\delta p^{\theta} \rightarrow \mathrm{v}$ and $\delta p^{\varphi} \rightarrow \mathrm{u}$, we obtain

$$
\begin{gathered}
\partial_{t} \delta \rho+\partial_{x} \mathrm{u}+\partial_{y} \mathbf{v}+\mathrm{v} \cot \theta_{0}=0, \\
\partial_{t} \mathrm{u}+\lambda p_{0} \partial_{x} \mathrm{u}+\frac{v_{1}}{R^{2} \sin ^{2} \theta_{0}} \partial_{x} \delta \rho \\
=p_{0}\left(a \delta \rho-2 b R^{2} p_{0} \sin ^{2} \theta_{0} \mathrm{u}\right)-\mathrm{v} \frac{\lambda p_{0}(\eta+2)}{2} \cot \theta_{0},
\end{gathered}
$$

$$
\partial_{t} \mathrm{v}+\lambda p_{0} \partial_{x} \mathrm{v}+\frac{v_{1}}{R^{2}} \partial_{y} \delta \rho=2 \mathrm{u} \lambda p_{0} \sin ^{2} \theta_{0} \cot \theta_{0}
$$

with $p_{0}=p_{s s}^{\varphi}\left(\theta_{0}\right)$ the azimuthal polarization at latitude $\theta_{0}$, which is finite as long as we are away from the poles, $\theta_{0} \neq 0, \pi$. Note that the stability of the steady state requires $\eta>0$, and hence $\lambda>0$. The only terms that can be negative are those proportional to $\cot \theta_{0}$, arising from the Christoffel symbols, which change sign as one crosses the equator at $\theta_{0}=\pi / 2$.

Next, we perform a Galilean boost to a comoving frame by letting $x \rightarrow x-\lambda p_{0} t$ (comoving with the longitudinal sound and not the flock itself), and relabel $\bar{\lambda}=\lambda p_{0}$, $\alpha=a p_{0}>0, \quad \beta=2 b p_{0}^{2} R^{2} \sin ^{2} \theta_{0}>0, \bar{v}_{1}=v_{1} / R^{2}>0$, and
TABLE I. A summary of the parameter redefinitions in the model.

\begin{tabular}{lccc}
\hline \hline$\lambda$ & $a$ & $b$ & $v_{1}$ \\
\hline $\bar{\lambda}=\lambda p_{0}$ & $\alpha=a p_{0}$ & $\beta=2 b p_{0}^{2} R^{2} \sin ^{2} \theta_{0}$ & $\bar{v}_{1}=v_{1} / R^{2}$ \\
\hline \hline & $m=-\cot \theta_{0}$.
\end{tabular}

The redefined parameters are summarized in Table I. Since the flock breaks Galilean invariance, this is not a symmetry operation, and it yields

$$
\begin{gathered}
\partial_{t} \delta \rho-\bar{\lambda} \partial_{x} \delta \rho+\partial_{x} \mathrm{u}+\partial_{y} \mathbf{v}=m \mathbf{v}, \\
\partial_{t} \mathrm{u}+\frac{\bar{v}_{1}}{\sin ^{2} \theta_{0}} \partial_{x} \delta \rho=\alpha \delta \rho-\beta \mathrm{u}+\frac{\bar{\lambda}(\eta+2)}{2} m \mathbf{v}, \\
\partial_{t} \mathbf{v}+\bar{v}_{1} \partial_{y} \delta \rho=-2 \bar{\lambda} \sin ^{2} \theta_{0} m \mathrm{u} .
\end{gathered}
$$

Here, $m$ is a constant of fixed sign at any given nonequatorial latitude and changes sign across the equator, with $m<0$ in the northern hemisphere and $m>0$ in the southern half, vanishing only at the equator where $\theta_{0}=\pi / 2$. We show below that a nonvanishing value of $m$ leads to a band gap [Figs. 3(b) and 3(c)] in the sound mode spectrum that acquires the necessary structure for nontrivial band topology. This, along with the vanishing of $m$ at the equator, naturally suggests that the equator behaves as a "boundary" between two different "bulk" media (the northern and southern hemispheres), thereby allowing for localized topologically protected excitations on it. To simplify the notation, we let $|\delta \Psi\rangle \equiv(\delta \rho, \mathrm{u}, \mathrm{v})$ and recast the linearized equations that control the linear stability of the steady state in the form of a Schrödinger-like equation [in Fourier space, with $\left.\Psi(\mathbf{q})=\int \mathrm{d}^{2} r e^{-i \mathbf{q} \cdot \mathbf{r}} \Psi(\mathbf{r})\right]$, as

$$
i \partial_{t}|\delta \Psi\rangle=H|\delta \Psi\rangle,
$$

$H(\mathbf{q})=\left(\begin{array}{ccc}-\bar{\lambda} q_{x} & q_{x} & i m+q_{y} \\ i \alpha+\frac{\bar{v}_{1} q_{x}}{\sin ^{2} \theta_{0}} & -i \beta & i m \bar{\lambda}\left(\frac{\eta}{2}+1\right) \\ \bar{v}_{1} q_{y} & -2 i m \bar{\lambda} \sin ^{2} \theta_{0} & 0\end{array}\right)$.

The eigenvalues of $H(\mathbf{q})$ directly give the sound mode frequencies $\left(|\delta \Psi\rangle \propto e^{-i \omega t}\right)$. An important distinction compared to the Schrödinger equation is that the matrix $H$ is not Hermitian, and therefore, the linearized mode spectrum is not purely real because of dissipative terms describing the overdamped dynamics and the absence of Galilean invariance. For $m=0\left(\theta_{0}=\pi / 2\right)$, the equations reduce to those 


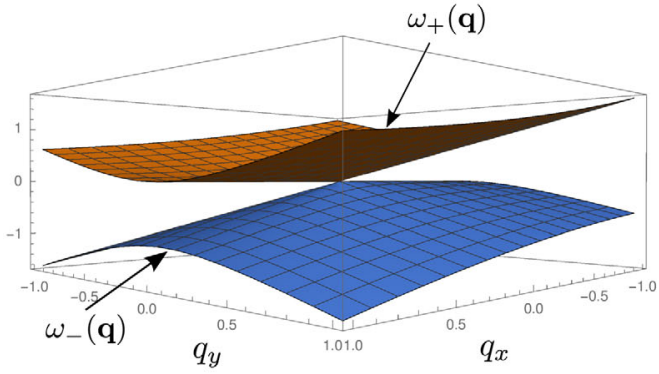

(a)

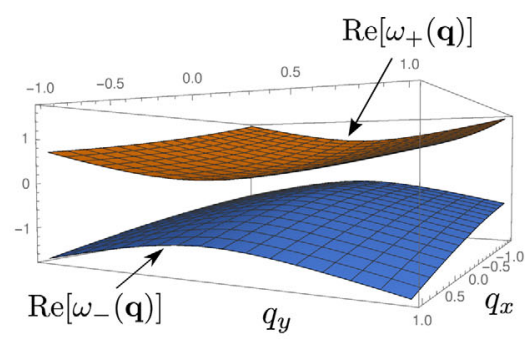

(b)

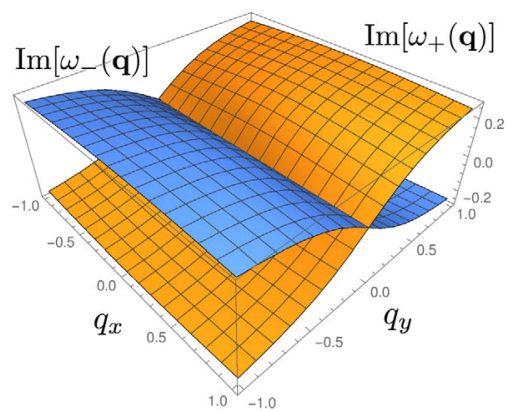

(c)

FIG. 3. The relevant (slow) sound modes. (a) $m=0$ and the gap between the two bands is closed. Panels (b) and (c) are the dispersion bands for $m=0.2$ [Eq. (25)], and we directly see that a gap has opened in the real part of the spectrum, while the imaginary part of the frequency has a single crossing line at $q_{y}=0$. The variables are chosen to be $\theta_{0} \simeq 78^{\circ}, \alpha \simeq 2.03, \bar{\lambda} \simeq 1.03$, and $\beta=\bar{v}_{1}=1$.

of the planar case. Fluctuations in the polarization magnitude (u) are controlled by a fast mode that decays on microscopic time scales $\sim \beta^{-1}$,

$$
i \omega_{0}(\mathbf{q})=\beta-i \frac{\alpha}{\beta} q_{x}+\mathcal{O}\left(q^{2}\right)
$$

The density $(\delta \rho)$ and the transverse Goldstone mode ( $\mathrm{v}$ ) are the only slow modes that remain propagating at long wavelengths (as $\mathbf{q} \rightarrow 0$ ),

$$
\omega_{ \pm}(\mathbf{q})=\frac{1}{2 \beta}\left[(\alpha-\beta \bar{\lambda}) q_{x} \pm \sqrt{(\alpha-\beta \bar{\lambda})^{2} q_{x}^{2}+4 \bar{v}_{1} \beta^{2} q_{y}^{2}}\right]
$$

where we have only kept terms to leading order in $\mathbf{q}$.

For nonzero but small $m \neq 0\left(\theta_{0} \neq \pi / 2\right)$, corresponding to the regions close to the equator in either hemisphere, the dispersion relations can be written as

$$
i \omega_{0}(\mathbf{q})=\beta-i \frac{\alpha}{\beta}\left(q_{x}+\frac{2 m \bar{\lambda}}{\beta} \sin ^{2} \theta_{0} q_{y}\right)+\mathcal{O}\left(q^{2}\right),
$$

In the next section, we analyze this mode structure.

\section{TOPOLOGICAL SOUND}

We immediately see that the two branches of the propagating modes given by Eq. (25) have a gap at $\mathbf{q}=0$ of width $\Delta=\left|\omega_{+}(0)-\omega_{-}(0)\right|$ proportional to $|m|$, with

$$
\Delta=2|m| \sin \theta_{0} \sqrt{\frac{2 \alpha \bar{\lambda}}{\beta}}+\mathcal{O}\left(m^{3}\right) .
$$

The terms explicitly involving $m$ in the dispersion relations, responsible for the opening of the gap, are obtained only in the presence of both curvature and spontaneous active flow. In the plane, static long-wavelength deformation of both the density and the broken symmetry mode leave the system unchanged. On a curved surface, in contrast, spatially uniform deformations of either "slow" field ( $\delta \rho$ and $v$ ) cannot be static and invariably lead to dynamics in the system. As a result of curvature-induced forces, long-wavelength deformations of would-be slow modes are required to have a finite frequency, resulting generically in the $\mathbf{q}=0$ gap of the sound spectrum, in sharp contrast to the conventional behavior of hydrodynamics in flat geometry [58].

It is useful to compare the effect at hand with one that occurs in geophysical flows. In a frame comoving with the flock, the finite curvature of the sphere plays a role similar to the Coriolis force that would be present for a passive fluid on a rotating sphere. In the case of the Earth's atmosphere, this has recently been shown to give a gapped sound spectrum and equatorially confined Kelvin and Yanai waves [59] that are topological in origin [39]. In our active system, no external flow or rotation needs to be imposed, and the absence of Galilean invariance allows for independent tuning of the material parameters (such as $\lambda$ ) in order to probe regimes that are not accessible to passive fluids.

On times scales $t \gg \beta^{-1}$, we can slave the fast mode $\mathrm{u}$ to the slow fields, $\mathrm{u} \simeq \alpha \delta \rho / \beta+\mathcal{O}(\nabla \delta \rho)$ [60]. Upon eliminating $\mathrm{u}$, we get a reduced set of dynamical equations 
involving only $\delta \rho$ and $\mathrm{v}$. After rescaling the wave vector $|\alpha \beta-\bar{\lambda}| q_{x} / \beta \rightarrow q_{x}$, the linear matrix controlling the dynamics of $\delta \rho, \mathrm{v}$ fluctuations is given by

$$
D(\mathbf{q})=\left(\begin{array}{cc}
s q_{x} & q_{y}+i m \\
\bar{v}_{1} q_{y}-i \mu m & 0
\end{array}\right),
$$

where $\mu=2 \bar{\lambda} \alpha \sin ^{2} \theta_{0} / \beta>0\left(\theta_{0} \neq 0, \pi\right)$ and $s=\operatorname{sgn}(\alpha-$ $\bar{\lambda} \beta)(s=0$, if $\bar{\lambda}=\alpha / \beta)$. One can easily check that the eigenfrequencies of $D(\mathbf{q})$ are exactly given by $\omega_{ \pm}(\mathbf{q})$ [Eq. (25)] modulo the appropriate rescaling of $q_{x}$. As $D(\mathbf{q})$ is still non-Hermitian, we need to evaluate right and left (adjoint) eigenvectors,

$$
\begin{gathered}
D(\mathbf{q})\left|\psi_{i}\right\rangle=\omega_{i}(\mathbf{q})\left|\psi_{i}\right\rangle, \\
D^{\dagger}(\mathbf{q})\left|\chi_{i}\right\rangle=\omega_{i}^{*}(\mathbf{q})\left|\chi_{i}\right\rangle \quad(i= \pm),
\end{gathered}
$$

with the biorthogonality relation $\left\langle\chi_{i} \mid \psi_{j}\right\rangle=\delta_{i j}$. It is important to keep in mind the regime in which $D(\mathbf{q})$ provides a valid approximation to the complete dynamics. For

$$
\frac{1}{\beta} \ll t \ll \frac{\beta}{\bar{\lambda}^{2} \eta m^{2}}, \quad \frac{\bar{\lambda}|m|}{\beta} \ll 1,
$$

we can neglect the fast $u$ mode and not worry about higherorder terms in both $\mathbf{q}$ and $m$. This can be achieved deep in the ordered phase on a large-enough sphere, in which case $\beta$ is large, allowing for a large window of time in which the dynamics is dominated by $D(\mathbf{q})$. With this set of simplifications, the linear dynamical matrix is always diagonalizable and $\omega_{+} \neq \omega_{-}$as long as $m \neq 0$ or $\mathbf{q} \neq 0$, allowing one to adiabatically deform our model to have purely real eigenvalues by smoothly taking $\mu \rightarrow \bar{v}_{1}$ (for $\mu \neq 0$ ). In the process, the spectral gap remains open as long as $m \neq 0$.

In order to establish the topological nature of the band structure, we compute the associated $\mathrm{U}(1)$ Berry gauge connection and curvature [61]

$$
\mathcal{A}_{ \pm}=i\left\langle\chi_{ \pm}\left|\nabla_{\mathbf{q}}\right| \psi_{ \pm}\right\rangle, \quad \mathcal{F}_{ \pm}(\mathbf{q})=\nabla_{\mathbf{q}} \times \mathcal{A}_{ \pm},
$$

along with the Chern numbers [62] for each band,

$$
C_{ \pm}=\int \frac{\mathrm{d}^{2} q}{2 \pi} \mathcal{F}_{ \pm}= \pm \frac{s}{2} \operatorname{sgn}(m),
$$

where $s=\operatorname{sgn}(\alpha-\bar{\lambda} \beta)$. The Chern number here is only quantized to a half integer as we work directly in the continuum long-wavelength approximation, and the closing of the gap (for $m=0$ ) only gives rise to one Diraccone-like structure (this is the contribution to the "parity anomaly" or Hall conductance associated with a single Dirac cone in a Chern insulator [63]). An appropriate regularization for large $\mathbf{q}$ guarantees the Chern number to be an integer $[38,64]$. However, this calculation still has worth in predicting the correct number of topologically protected edge modes present when we stitch two of these regions with different Chern numbers together, via the bulk-edge correspondence. So each gap closing (change in sign of $m$ ) leads to a single localized edge mode, which, as we shall see, is unidirectional.

As anticipated earlier, we find that the Chern number of the acoustic band is different in the northern $(m<0)$ and southern $(m>0)$ hemispheres, vanishing at the equator $(m=0)$. Hence, going across the equator, we have one gap closing (at $\mathbf{q}=0$ ) with a band inversion, leading to a single topological sound excitation localized at the equator. Note that the Chern number also vanishes for $s=0$, which is obtained either when $p_{0}=0$ or $\bar{\lambda}=\alpha / \beta$, leading to a topologically trivial band structure. The first case corresponds to the absence of spontaneous active flow and the second to a partial restoration of Galilean invariance (in this limit, both density and Goldstone mode excitations propagate with the same longitudinal speed). Thus, the vanishing of the Chern number and associated band triviality for $s=0$ is not due to the closure of a gap but instead to a restoration of symmetry. As $\beta=2 b R^{2} p_{0}^{2} \sin ^{2} \theta_{0}$ depends on the latitude at which we are located, the vanishing of $s$ (for $\bar{\lambda}=\alpha / \beta$ ) is a condition on the polar angle $\theta_{0}$. There is a critical density

$$
\rho^{*}=\rho_{c}+\frac{1}{2 A_{\eta} \lambda},
$$

such that for $\rho_{c}<\rho_{0}<\rho^{*}, s \neq 0$ on the entire sphere. Deeper into the ordered state $\left(\rho_{0}>\rho^{*}\right)$, there are two latitudes at angles $\theta_{ \pm}$such that $\sin ^{\eta} \theta_{ \pm}=$ $\left(\rho^{*}-\rho_{c}\right) /\left(\rho_{0}-\rho_{c}\right)$, at which $s=0$. Even though the band topology changes as we cross the latitudes at $\theta_{ \pm}$[Eq. (33)], the spectrum remains gapped throughout, and hence we $d o$ not have any gapless excitations localized at $\theta_{ \pm}$. The change in the Chern number across these special latitudes is due to an accidental additional symmetry (Galilean invariance) instead of the gap closing, thereby circumventing the bulk-boundary correspondence. This is a wellknown point in quantum topological insulators, only realized here in a peculiar fashion as the protecting "symmetry" varies spatially in a single sample.

To summarize, there are three crucial ingredients in this system that lead to and protect the topologically nontrivial band structure.

(i) Breaking of time-reversal symmetry by the active polar flow. Changing the direction of spontaneous polarization (flow) changes the sign of $s$ (as $p_{0} \rightarrow$ $-p_{0}$ leads to $\alpha \rightarrow-\alpha, \bar{\lambda} \rightarrow-\bar{\lambda}, \beta \rightarrow \beta$, and $\mu \rightarrow \mu$ ).

(ii) The presence of the convective nonlinearity $\lambda \neq 0$. An equilibrium passive polar liquid crystal will therefore not exhibit these modes.

(iii) The curvature of the base surface, which opens up a gap in the sound spectrum. Changing the Gaussian 

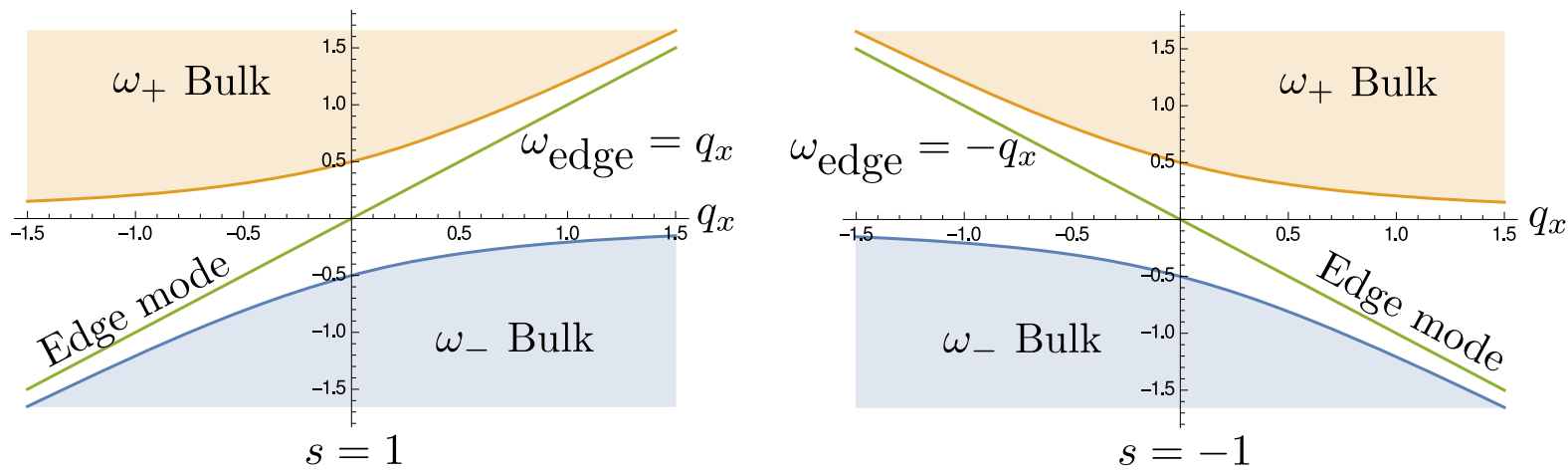

FIG. 4. The bulk and edge mode spectrum for the case when $s= \pm 1$ (shown for the simple case when $\left.\mu=\bar{v}_{1}\right)$ and $m(y)$ varying from -1 to +1 .

curvature exchanges the regions with positive and negative " $m$ ".

This is entirely analogous to the Haldane model [63], where the closing of the gap at the Dirac point is protected by time-reversal symmetry, which, when broken by the local magnetic field, leads to a band structure with a nontrivial topology. Though the active system is not Hermitian with purely real frequencies, the structure of the localized equatorial mode for varying $m(y)$ is adiabatically connected to its Hermitian analogue [65], the Jackiw-Rebbi soliton [66],

$$
\left|\delta \Psi_{\text {edge }}\right\rangle=\psi_{0} e^{-\eta \int_{0}^{y} m\left(y^{\prime}\right) \mathrm{d} y^{\prime}+i q_{x}(x-s t)}\left(\begin{array}{l}
1 \\
0
\end{array}\right)
$$

where $\psi_{0}$ is a normalization constant. The edge mode spectrum $\omega_{\text {edge }}\left(q_{x}\right)=s q_{x}$ corresponds to a pure one-way density wave that connects the two bulk bands (see Fig. 4). This edge mode is valid when $m(y) \rightarrow \pm m_{0}\left(m_{0}>0\right)$ for $y \rightarrow \pm \infty$.

On the sphere, reverting back to angular coordinates $\{\theta, \varphi\}, m(\theta)=-\cot \theta$, which is positive in the southern hemisphere for $\theta>\pi / 2(y>0)$. This gives a chiral equatorial density mode $(\mathbf{v}=0)$, which in the lab frame looks like

$$
\delta \rho_{\text {edge }}(\theta, \varphi ; t)=\sin ^{\eta} \theta \sum_{n \geq 0}\left[\mathrm{a}_{n} e^{i n(\varphi-\alpha t / \beta)}+\text { c.c. }\right],
$$

where $\mathrm{a}_{n}$ are complex constants depending on the initial perturbation applied. A snapshot of this density mode is shown in Fig. 6 for $n=6$ (and all other $\mathrm{a}_{n}$ vanishing). Equation (36) defines a localization length $\ell_{\mathrm{loc}}=R / \eta$ set by the curvature and material parameters of the active fluid and essentially given by the ratio of longitudinal to transverse sound speeds. Note that the result given in Eqs. (35) and (36) applies for $\lambda_{3}=0$. The general case of $\lambda_{2}, \lambda_{3} \neq 0$ is given in Appendix A and yields a different localization width for the equatorial mode. This topological edge mode propagates unidirectionally in the direction of the flock and is robust to disorder because there are no reverse channels into which it can scatter (though it will eventually dissipate because of viscous and elastic damping). Unlike a Galilean invariant fluid for which $\eta=1$, here $\eta$ and therefore the localization length can be tuned by varying the system's parameters, although the shape of the steady-state profile remains unchanged.

\section{POLAR FLOCK ON A NEGATIVE CURVATURE SURFACE}

The presence of such topological excitations is generic in that they will always be present when one has a polar flock on a curved surface that looks locally like a surface of revolution [67]. We illustrate this point on a catenoid, a surface with nonconstant negative Gaussian curvature.

In local coordinates $\{y, \varphi\}$ ( $\varphi$ once again being the periodic azimuthal direction), the metric and Gaussian curvature on a catenoid are

$$
\begin{gathered}
\mathfrak{g}=R^{2} \cosh ^{2} y(\mathrm{~d} y \otimes \mathrm{d} y+\mathrm{d} \varphi \otimes \mathrm{d} \varphi), \\
K_{G}(y)=-\frac{1}{R^{2}} \operatorname{sech}^{4} y,
\end{gathered}
$$

where $R$ is the radius of curvature at the neck of the catenoid. In contrast to the sphere, the Gaussian curvature here is both negative and spatially varying. The only nonvanishing Christoffel symbols are

$$
\Gamma_{\varphi y}^{\varphi}=\Gamma_{y y}^{y}=-\Gamma_{\varphi \varphi}^{y}=\tanh y .
$$

Taking the same approach as for the sphere, neglecting viscous and elastic stresses, we consider an azimuthally symmetric ansatz for the steady-state polar flock: $\rho=\rho_{s s}(y), p^{y}=0$, and $p^{\varphi}=p_{s s}^{\varphi}(y)$. One can easily verify that for $\rho_{0}>\rho_{c}$, the steady-state density profile is then 


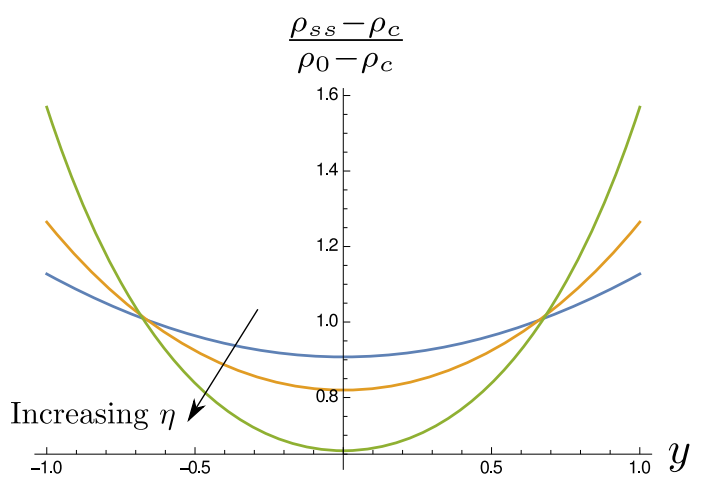

(a)

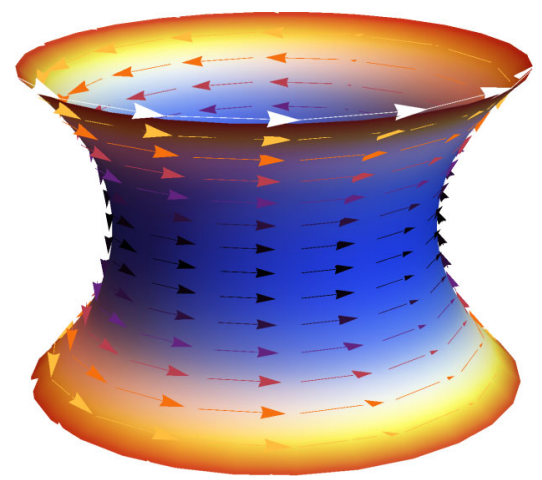

(b)

FIG. 5. (a) The normalized density profile of a polar flock on a catenoid given in Eq. (40), for $\eta=0.5$ (blue line), 1 (orange line), and 2 (green line). Note that, unlike the sphere, the density grows near the edge of the catenoid. (b) The density and polarization (for $\eta=2$ ), now shown on the catenoid. As before, blue corresponds to low-density regions (at the neck) and red to high-density regions.

$$
\rho_{s s}(y)=\rho_{c}+\left(\rho_{0}-\rho_{c}\right) B_{\eta} \cosh ^{\eta} y
$$

where $B_{\eta}<1$ is a constant that depends on $\eta$ and the height of the catenoid (which, unlike the sphere, is not compact and has to be taken finite). The details of the computation are given in Appendix B. In contrast to the sphere, which had a polar band with maximum density at the equator, the polar flock density is lowest at the neck of the catenoid $(y=0)$, increasing on either side as one moves away from it. The corresponding polarization profile is given by

$$
\left|\mathbf{p}_{s s}\right|^{2}=\frac{a\left(\rho_{0}-\rho_{c}\right)}{b} B_{\eta} \cosh ^{\eta} y .
$$

The density and polarization profiles are plotted in Fig. 5(a). Below the mean-field transition $\left(\rho_{0}<\rho_{c}\right)$, we recover the isotropic disordered phase $\left(\rho_{s s}=\rho_{0}, \mathbf{p}_{s s}=\mathbf{0}\right)$.

Linearizing about this steady state, one finds that the equations governing the propagation of sound modes on the catenoid are essentially identical to that on the sphere [Eqs. (14)-(16)] but with modified parameters. As a
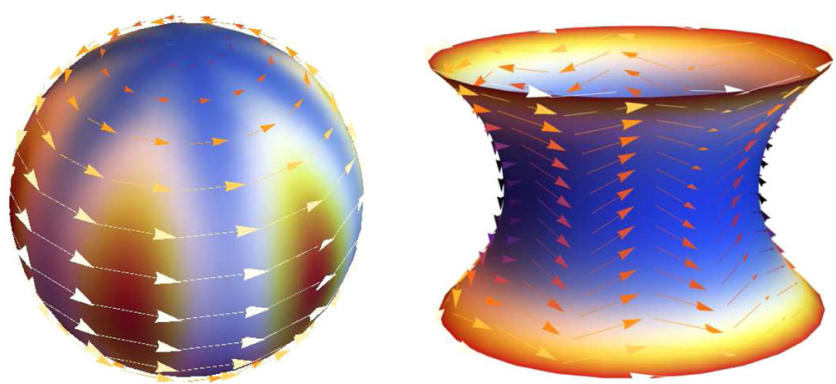

FIG. 6. A representative snapshot of the equatorial density mode on a sphere (Eq. (36)) and the localized Goldstone mode on the catenoid (Eq. (42)). For clear visualization we have chosen the perturbation $\mathrm{a}_{6}, \mathrm{~b}_{6}=0.5$ and all other an, $\mathrm{b}_{n}=0(n \neq 6)$. We have also taken both $\eta=\mu / \bar{v}_{1}=2$ both cases.

consequence of the negative curvature, the most important change is that $m=-2 \tanh y$ is positive below the neck of the catenoid $(y<0)$ and negative above $(y>0)$, vanishing right at the neck $(y=0)$. This leads to a chiral mode of Goldstone fluctuations localized at the neck of the catenoid $(\delta \rho=0)$, which, written in the lab frame, is given by

$$
\delta \mathrm{v}_{\text {edge }}(\varphi, y ; t)=\operatorname{sech}^{2} y \sum_{n \geq 0}\left[\mathrm{~b}_{n} e^{i n(\varphi-\bar{\lambda} t)}+\text { c.c. }\right]
$$

where, as before, $\bar{\lambda}=\lambda p_{0}$ and $\mathrm{b}_{n}$ is a complex coefficient determined by the initial perturbation. A snapshot of this mode is shown as well in Fig. 6. This mode propagates in the same direction as the flock but with a different speed, and it is topologically protected. The localization length $\ell_{\mathrm{loc}}=2 R$ is controlled by the scale of the curvature in the system and seemingly independent of the material parameters of the flock.

\section{CONCLUSION}

The frustration associated with the interplay of curvature and order $[30,68,69]$ has many consequences for crystals [70-74], tethered membranes [75,76], liquid crystalline membranes [77-79], and jammed and glassy systems $[80,81]$. The presence of activity adds an entirely new nonequilibrium dimension to the whole story, allowing for completely new physics arising from competing order, curvature, and the active drive. The active polar fluid is peculiar, as the polarization order parameter is also a velocity that advects the fluid [4], with this dual role being at the heart of many of the phenomena we have explored in this article. In particular, unlike a superfluid film on a curved substrate (where the order parameter can be parallel transported trivially) [82], the fact that the order parameter of the flock is a physical velocity implies that it advects itself nontrivially in the presence of curvature. This is nothing but a restatement of the physical fact that selfpropelled particles move persistently along geodesics (in 
the absence of interactions), which get "lensed" by curvature, whereas passive polar particles do not.

In order to handle these points, we generalized the continuum Toner-Tu model for an active polar fluid to an arbitrary curved surface and found new terms that are absent in flat space. In general, studying ordering phenomena on curved surfaces is rather complicated, even in equilibrium. This is when symmetry is often a very useful guiding tool, by which we explicitly computed the ordered phase of an active polar flock on two surfaces, the sphere and the catenoid. The continuum model affords us the privilege of having model-independent predictions, and we find that many of the features of the steady ordered state of a polar flock are quite generic, with positive curvature surfaces having density profiles with a maximum, decreasing on either side of the peak, while negative curvature surfaces have the density profiles of the opposite kind, being minimal in the interior and increasing towards the boundary. Finding such spatially inhomogeneous exact solutions to the covariant Toner-Tu model is definitely a crucial starting point to being able to understand how the phenomenology of active matter in flat space translates to its curved variants.

In addition to the steady state with spontaneous flow, flocks in flat space also have dissipative sound modes with a linear (but angle-dependent) dispersion [42]. It is here that all three players-curvature, order, and activity-come together with dramatic consequences. The presence of curvature gaps the sound mode spectrum at long wavelengths, leading to a band structure with nontrivial topology protected by the broken time-reversal and Galilean symmetry in the system. We demonstrate this by computing the Chern number for the bands and show that this is a generic feature of flocks on curved surfaces. The most interesting result of the nontrivial band topology is to localize "edge" modes of density or Goldstone mode fluctuations along special geodesics on the surface, at which the gap in the sound spectrum vanishes. The rather novel feature here is that the system is not artificially engineered as a metamaterial with some underlying lattice structure $[33,83]$, nor does it require any external force or fields of any kind [34]. The spontaneous flow is generated by activity breaking both time-reversal and Galilean invariance simultaneously, while the curvature is responsible for the spectral gap in the ordered phase.

Topological excitations of the type described here are "protected" in the sense that they are robust against static perturbations and heterogeneities in the medium through which they propagate. While quantifying the limits of such topological protection in active systems will require numerical work and remains to be explored, our work demonstrating that such topologically protected propagating modes are a generic consequence of active flows on curved surfaces raises the question of whether nature may use this mechanism to guide and direct the robust transmission of intercellular physical forces in curved environments. It is therefore tempting to offer some speculation to the possible relevance of our findings to biology. In a number of developmental phenomena, from wound healing to morphogenesis and organ development, living cells migrate collectively, offering an intriguing realization of a polar active fluid. While a full understanding of the mechanisms that regulate collective cell migration is still out of reach, it is now widely recognized that the transmission of physical forces plays an important role, alongside biochemical signaling. For instance, propagating mechanical waves have been shown to mediate cooperative force transmission among epithelial cells in wound-healing assays [84]. In many biological processes, cell motion takes place on curved surfaces, as in cell renewal and repair in the highly folded intestine [85] and the shaping of the early limb bud in developing embryos [86]. The effect of curvature on the dynamics of epithelial cells is beginning to be explored in vitro by examining collective cell migration on cylindrical capillaries of varying radii [21]. While cylinders have zero Gaussian curvature, which would not yield topologically protected states, these experiments clearly demonstrate that curvature affects cell morphology and dynamics by enhancing cell speed and cell extrusion. A more direct application of the work described here would be to a polar version of the active nematic vesicles described in Ref. [10]. Here, active vesicles were engineered by confining an active suspension of microtubule-kinesin bundles to the surface of a lipid vesicle. The interplay of activity and curvature yields a number of dynamical structures, including spontaneously oscillating defect textures and folding nematic bands and, ultimately, activity-driven shape deformations of the vesicle. Our work may also be relevant to the physics of cell membranes that are activated through coupling to the polymerizing acto-myosin cortex, as modeled in recent work by Maitra et al. [31]. Finally, in the spirit of colloidal crystals on curved interfaces and reconstituted active systems, one might also envision synthetic experimental realizations of the topological sound modes investigated here, by depositing active Janus colloidal rods or active biofilament motor complexes on the surface of a droplet or a vesicle shell.

\section{ACKNOWLEDGMENTS}

We thank Brad Marston for inspiring this work through a beautiful talk delivered at KITP based on Ref. [39]. We also thank Rastko Sknepnek and Vladimir Juričić for useful discussions. This work was supported by the National Science Foundation at Syracuse University through Grants No. DMR-1609208 (M. C. M. and S.S.) and DGE1068780 (M. C. M.) and at KITP under Grant No. NSF PHY-1125915. All authors thank the Syracuse Soft Matter Program for support and the KITP for its hospitality during the completion of some of the work. 


\section{APPENDIX A: STEADY STATE AND LINEARIZATION FOR $\lambda_{2}, \lambda_{3} \neq 0$}

Including the two additional $\lambda_{2} \mathbf{p} \nabla \cdot \mathbf{p}$ and $\lambda_{3} \nabla|\mathbf{p}|^{2}$ nonlinearities, the equation for the polarization order parameter is modified to

$$
\begin{aligned}
\partial_{t} p^{\mu}+\lambda p^{\nu} \nabla_{\nu} p^{\mu}= & {\left[a\left(\rho-\rho_{c}\right)-b g_{\alpha \beta} p^{\alpha} p^{\beta}\right] p^{\mu} } \\
& +\lambda_{2} p^{\mu} \nabla_{\nu} p^{\nu}+\lambda_{3} \nabla^{\mu}\left(p^{\nu} p_{\nu}\right)-v_{1} \nabla^{\mu} \rho .
\end{aligned}
$$

We do not include the viscous terms $\nu, \nu^{\prime}$, consistent with our approximations in the main text. For the azimuthally symmetric ansatz (on the sphere and the catenoid), $\nabla_{\mu} p_{s s}^{\mu}=0$ identically, and hence the $\lambda_{2}$ terms do not affect the steady-state profile. On the contrary, the $\lambda_{3} \nabla|\mathbf{p}|^{2}$ term acts as an additional polarization-dependent contribution to the scalar pressure $P \sim v_{1} \rho-\lambda_{3}|\mathbf{p}|^{2}$, which, if large, can lead to density and splay instabilities [49]. We disregard this and only work in the regime where $\lambda_{3}$ is not large enough to destabilize the entire system. Including it, the steady-state equations on the sphere [Eqs. (6) and (7)] get modified to

$$
\begin{gathered}
\lambda \sin \theta \cos \theta\left(p_{s s}^{\varphi}\right)^{2}=\frac{v_{1}}{R^{2}} \partial_{\theta} \rho_{s s}-\lambda_{3} \partial_{\theta}\left(\sin ^{2} \theta\left(p_{s s}^{\varphi}\right)^{2}\right), \\
p_{s s}^{\varphi}\left[a\left(\rho_{s s}-\rho_{c}\right)-2 b R^{2} \sin ^{2} \theta\left(p_{s s}^{\varphi}\right)^{2}\right]=0 .
\end{gathered}
$$

Once again, setting $X(\theta)=\rho_{s s}(\theta)-\rho_{c}$, we get the same equation as before, only now with a modified coefficient depending on $\lambda_{3}$,

$\frac{\mathrm{d} X}{\mathrm{~d} \theta}=\frac{\lambda a}{v_{1} b-a \lambda_{3}} \cot \theta X \Rightarrow X(\theta)=X(\pi / 2) \sin ^{\eta^{\prime}} \theta$,

with the exponent now changed to $\eta^{\prime}=\lambda a /\left(v_{1} b-a \lambda_{3}\right)$. For $\eta^{\prime}>0$ (to have a physical density profile), we require $v_{1} b>a \lambda_{3}$, which is nothing but a condition to have stable pressure and a positive compressibility. Hence, the effect of $\lambda_{3} \neq 0$ is to only change the density profile through the $\eta$ exponent, with the functional form remaining the same. This is true even for the catenoid, where the exponent is the same as on the sphere and given by $\eta^{\prime}=\lambda a /\left(v_{1} b-a \lambda_{3}\right)$. Note that this inhomogeneous profile does not exist for an equilibrium polar liquid crystal for which $\lambda=0$ (but possibly $\lambda_{2}, \quad \lambda_{3} \neq 0$, allowing for spontaneous splay [56]), leading to $\eta^{\prime}=0$. Hence, the inhomogeneous steady state we obtain is only possible in an active system.

Linearizing about the steady state (on the sphere), now including the $\lambda_{2}$ and $\lambda_{3}$ terms, we get

$$
\partial_{t} \delta \rho+\partial_{x} \mathbf{u}+\partial_{y} \mathbf{v}=m \mathbf{v}
$$

$$
\begin{gathered}
\partial_{t} \mathrm{u}+\left(\bar{\lambda}-\bar{\lambda}_{2}-2 \bar{\lambda}_{3}\right) \partial_{x} \mathrm{u}+\frac{\bar{v}_{1}}{\sin ^{2} \theta_{0}} \partial_{x} \delta \rho \\
=\alpha \delta \rho-\beta \mathrm{u}+\mathbf{v} m\left(\bar{\lambda} \frac{\eta^{\prime}+2}{2}-\bar{\lambda}_{2}\right)+\bar{\lambda}_{2} \partial_{y} \mathbf{v}, \\
\partial_{t} \mathbf{v}+\bar{\lambda} \partial_{x} \mathrm{u}+\bar{v}_{1} \partial_{y} \delta \rho= \\
-\left(2 \bar{\lambda}+\left(4+\eta^{\prime}\right) \bar{\lambda}_{3}\right) \sin ^{2} \theta_{0} m \mathrm{u} \\
+2 \bar{\lambda}_{3} \sin ^{2} \theta_{0} \partial_{y} \mathrm{u} .
\end{gathered}
$$

We use the same notation as we used in the main text (along with $\left.\bar{\lambda}_{2,3}=\lambda_{2,3} p_{0}\right)$. At long times $\left(t \gg \beta^{-1}\right)$, the polarization magnitude $u$ is still a fast mode, and to leading order, it gets slaved to the density fluctuations in the same fashion as before $(\mathrm{u} \simeq \alpha \delta \rho / \beta)$. Consistent with the level of approximation used in the main text [Eq. (31)], the long-time and long-wavelength dynamics are governed only by the two slow modes $\delta \rho$ and $\mathrm{v}$, with a dynamical matrix $D(\mathbf{q})$ of the same form as found in the absence of $\lambda_{2}$ and $\lambda_{3}$,

$$
D(\mathbf{q})=\left(\begin{array}{cc}
s q_{x} & q_{y}+i m \\
\bar{v}_{1}^{\prime} q_{y}-i \mu^{\prime} m & 0
\end{array}\right),
$$

with $s=\operatorname{sgn}(\alpha-\bar{\lambda} \beta)$ and the only modification being in the coefficients $\bar{v}_{1}^{\prime}$ and $\mu^{\prime}$,

$$
\bar{v}_{1}^{\prime}=\bar{v}_{1}-2 \bar{\lambda}_{3} \sin ^{2} \theta_{0} \frac{\alpha}{\beta}=\frac{v_{1} b-a \lambda_{3}}{b R^{2}},
$$

$$
\mu^{\prime}=\left(2 \bar{\lambda}+\left(4+\eta^{\prime}\right) \bar{\lambda}_{3}\right) \sin ^{2} \theta_{0} \frac{\alpha}{\beta}=a \frac{\left[2 \lambda+\left(4+\eta^{\prime}\right) \lambda_{3}\right]}{2 b R^{2}} .
$$

It is easy to see now that the profile of the localized equatorial density mode on the sphere, which was $\propto \sin ^{\mu^{\prime} / \bar{v}_{1}^{\prime}} \theta \quad$ [for $\quad \lambda_{2,3}=0, \quad \mu / \bar{v}_{1}=\eta \quad$ as given in Eq. (36)] is no longer the same as the steady-state density profile of the flock $\left(\sim \sin ^{\eta^{\prime}} \theta\right)$, when $\lambda_{3} \neq 0$,

$$
\frac{\mu^{\prime}}{\bar{v}_{1}^{\prime}}=\eta^{\prime}\left[1+\left(2+\frac{\eta^{\prime}}{2}\right) \frac{\lambda_{3}}{\lambda_{1}}\right] \neq \eta^{\prime} .
$$

A similar result also holds true for the catenoid. Hence, we find that, even upon including additional convective nonlinearities (which are lower order in gradients compared to the viscous terms), all of the qualitative properties of the steady state and the topologically protected modes remain the same, with the only modification being a more detailed dependence of the localization length on some of the material parameters in the system. 


\section{APPENDIX B: POLAR FLOCK OF THE CATENOID}

For an azimuthally symmetric ordered steady state on the catenoid, just as on the sphere, we neglect the viscous and elastic stresses, and use the ansatz: $\rho=\rho_{s s}(y), p^{y}=0$ and $p^{\varphi}=p_{s s}^{\varphi}(y)$. Plugging this into Eqs. (1) and (3), we find that the continuity equation is satisfied identically, and Eq. (3) reduces to (for $\nu=0$ )

$$
\begin{gathered}
\lambda \tanh y\left(p_{s s}^{\varphi}\right)^{2}=\frac{v_{1}}{R^{2} \cosh ^{2} y} \partial_{y} \rho_{s s}, \\
p_{s s}^{\varphi}\left[a\left(\rho_{s s}-\rho_{c}\right)-b R^{2} \cosh ^{2} y\left(p_{s s}^{\varphi}\right)^{2}\right]=0 .
\end{gathered}
$$

Setting $X(y)=\rho_{s s}(y)-\rho_{c}$, we solve the equations in the same fashion as before to get $\partial_{y} X=\eta \tanh y X$, where $\eta=\lambda a / b v_{1}$ (the same exponent as on the sphere). The steady-state density profile is then

$$
\rho_{s s}(y)=\rho_{c}+\left(\rho_{\min }-\rho_{c}\right) \cosh ^{\eta} y,
$$

where $\rho_{\min }$ is the minimum density of the flock attained on the neck of the catenoid $(y=0)$. Unlike the sphere, the catenoid is not a compact surface, so in reality, one would have a finite sample with boundaries. The mean density $\rho_{0}$ is given by the spatial average of the steady-state profile,

$$
\rho_{0}=\rho_{c}+\left(\rho_{\min }-\rho_{c}\right)\left\langle\cosh ^{\eta} y\right\rangle,
$$

where $\langle\cdot\rangle$ denotes a spatial average over the entire surface. For a catenoid of height $L$ (Euclidean height in the $z$ direction when embedded in $\mathbb{R}^{3}$ ) and radius of curvature $R$ at the minimal neck, we have

$$
\left\langle\cosh ^{\eta} y\right\rangle \simeq \begin{cases}1+\frac{\eta}{6}\left(\frac{L}{R}\right)^{2} & L / R \ll 1 \\ \frac{2^{1-\eta}}{2+\eta} e^{\eta L / R} & L / R \gg 1\end{cases}
$$

Writing $B_{\eta}=1 /\left\langle\cosh ^{\eta} y\right\rangle<1$, we obtain the density profile quoted in the main text [Eq. (40)].

We expect the viscous and elastic stresses to be less important on a weakly curved surface close to the neck, in particular, when the characteristic scale of curvature $(\sim R)$ is much greater than the equilibrium correlation length $\left[\xi \sim \sqrt{\nu / a\left(\rho_{0}-\rho_{c}\right)}\right]$. Additionally, the density and polarization (along with their gradients) grow larger as we go away from the neck. So, close to the boundaries of a large sample, one would have to account for higher-order nonlinearities along with the elastic stresses, which would then become important.

\section{Linearizing about the steady state}

One can perform the same kind of analysis as we did before for the flock on a sphere. Linearizing about the ordered flock, $\rho=\rho_{s s}(y)+\delta \rho$ and $\mathbf{p}=\mathbf{p}_{s s}(y)+\delta \mathbf{p}$ within the tangent plane at a distance $y_{0}$ from the $y=0$ neck, we get (with $x=\varphi, \mathrm{u}=\delta p^{\varphi}$, and $\mathrm{v}=\delta p^{y}$ just as before)

$$
\begin{gathered}
\partial_{t} \delta \rho+\partial_{x} \mathrm{u}+\partial_{y} \mathrm{v}+2 \mathrm{v} \tanh y_{0}=0 \\
\partial_{t} \mathrm{u}+\lambda p_{0} \partial_{x} \mathrm{u}+\frac{v_{1}}{R^{2} \cosh ^{2} y_{0}} \partial_{x} \delta \rho \\
=p_{0}\left(a \delta \rho-2 b R^{2} p_{0} \cosh ^{2} y_{0} \mathrm{u}\right)-\mathrm{v} \frac{\lambda p_{0}(\eta+2)}{2} \tanh y_{0},
\end{gathered}
$$

$\partial_{t} \mathbf{v}+\lambda p_{0} \partial_{x} \mathbf{v}+\frac{v_{1}}{R^{2} \cosh ^{2} y_{0}} \partial_{y} \delta \rho=2 \mathrm{u} \lambda p_{0} \tanh y_{0}$,

where $p_{0}=p_{s s}^{\varphi}\left(y_{0}\right)$. Galilean boosting to a moving frame $x \rightarrow x-\lambda p_{0} t$ and relabeling our parameters as before, we obtain $\bar{\lambda}=\lambda p_{0}, \alpha=a p_{0}>0, \beta=2 b R^{2} p_{0}^{2} \cosh ^{2} y_{0}>0$, $\bar{v}_{1}=v_{1} /\left(R^{2} \cosh ^{2} y_{0}\right)$, and $m=-2 \tanh y_{0}$. Having done this, all the arguments used in the case of the sphere apply here as well.

At long times $\left(t \gg \beta^{-1}\right)$, the fast polarization magnitude $\mathrm{u}$ decays and is slaved to the density field $\mathrm{u} \simeq \alpha \delta \rho / \beta$ (to lowest order), and the slow dynamics at long wavelengths is dominated by

$$
\begin{gathered}
\partial_{t} \delta \rho+(\alpha / \beta-\bar{\lambda}) \partial_{x} \delta \rho+\partial_{y} \mathbf{v}=m \mathbf{v}, \\
\partial_{t} \mathbf{v}+\bar{v}_{1} \partial_{y} \delta \rho=-\mu m \delta \rho,
\end{gathered}
$$

only now with $\mu=\alpha \bar{\lambda} / \beta>0$. Hence, at the same level of approximation used earlier for the sphere [neglecting viscous stresses and the parameter regime given in Eq. (31)], the long-time dynamics of sound excitations in a polar flock on a curved surface is generically described by equations of the form given above or, consequently, by the linear dynamical matrix $D(\mathbf{q})$ [Eq. (28)], possibly up to some coordinate rescaling.

As the only modifications are in the definitions of the parameters, many of the predictions made in the case of the sphere apply here, too. In particular, the sound mode spectrum is still gapped at $\mathbf{q}=0$ for nonzero $m$, and the bands have a nontrivial topology given by the Chern numbers $C_{ \pm}$[see Eq. (33)]. As $m=0$ at the neck of the catenoid $(y=0)$, changing sign on either side, we have one topologically protected mode localized at the neck. A consequence of the negative curvature of the surface is that, in contrast to the sphere, $m<0$ for $y>0$. Therefore, the edge mode takes on a different form (in the comoving frame),

$$
\left|\delta \Psi_{\text {edge }}\right\rangle=\psi_{0} e^{\int_{0}^{y} m\left(y^{\prime}\right) \mathrm{d} y^{\prime}+i q_{x} x}\left(\begin{array}{l}
0 \\
1
\end{array}\right) .
$$

Now, the edge mode is a localized mode of transverse Goldstone fluctuations, with density fluctuations completely 
absent. Additionally, the edge mode spectrum is $\omega_{\text {edge }}=0$ to lowest order in $q_{x}$, implying that the edge mode is stationary in the comoving (with speed $\sim \lambda p_{0}$ ) frame. This also connects the two bulk bands and is topologically protected. Using $m(y)=-2 \tanh y$ for the catenoid, this gives the profile of the localized mode, the lab frame version of which is quoted in the main text [Eq. (42)].

[1] T. Vicsek, A. Czirók, E. Ben-Jacob, I. Cohen, and O. Shochet, Novel Type of Phase Transition in a System of SelfDriven Particles, Phys. Rev. Lett. 75, 1226 (1995).

[2] J. Toner, Y. Tu, and S. Ramaswamy, Hydrodynamics and Phases of Flocks, Ann. Phys. (Amsterdam) 318, 170 (2005).

[3] S. Ramaswamy, The Mechanics and Statistics of Active Matter, Annu. Rev. Condens. Matter Phys. 1, 323 (2010).

[4] M. Cristina Marchetti, J. F. Joanny, S. Ramaswamy, T. B. Liverpool, J. Prost, M. Rao, and R. A. Simha, Hydrodynamics of Soft Active Matter, Rev. Mod. Phys. 85, 1143 (2013).

[5] A. Cavagna, A. Cimarelli, I. Giardina, G. Parisi, R. Santagati, F. Stefanini, and M. Viale, Scale-Free Correlations in Starling Flocks, Proc. Natl. Acad. Sci. U.S.A. 107, 11865 (2010).

[6] A. Sokolov, I. S. Aranson, J. O. Kessler, and R. E. Goldstein, Concentration Dependence of the Collective Dynamics of Swimming Bacteria, Phys. Rev. Lett. 98, 158102 (2007).

[7] B. Szabo, G. J. Szöllösi, B. Gönci, Z. Jurányi, D. Selmeczi, and T. Vicsek, Phase Transition in the Collective Migration of Tissue Cells: Experiment and Model, Phys. Rev. E 74, 061908 (2006).

[8] V. Schaller, C. Weber, C. Semmrich, E. Frey, and A. R. Bausch, Polar Patterns of Driven Filaments, Nature (London) 467, 73 (2010).

[9] T. Sanchez, D. T. N. Chen, S. J. DeCamp, M. Heymann, and Z. Dogic, Spontaneous Motion in Hierarchically Assembled Active Matter, Nature (London) 491, 431 (2012).

[10] F. C. Keber, E. Loiseau, T. Sanchez, S. J. DeCamp, L. Giomi, M. J. Bowick, M. C. Marchetti, Z. Dogic, and A. R. Bausch, Topology and Dynamics of Active Nematic Vesicles, Science 345, 1135 (2014).

[11] J. Deseigne, O. Dauchot, and H. Chaté, Collective Motion of Vibrated Polar Disks, Phys. Rev. Lett. 105, 098001 (2010).

[12] V. Narayan, S. Ramaswamy, and N. Menon, Long-Lived Giant Number Fluctuations in a Swarming Granular Nematic, Science 317, 105 (2007).

[13] J. Palacci, S. Sacanna, A. P. Steinberg, D. J. Pine, and P. M. Chaikin, Living Crystals of Light-Activated Colloidal Surfers, Science 339, 936 (2013).

[14] S. J. Streichan, M. F. Lefebvre, N. Noll, E. F. Wieschaus, and B. I. Shraiman, Quantification of Myosin Distribution Predicts Global Morphogenetic Flow in the Fly Embryo, arXiv:1701.07100.

[15] B. Vasiev, A. Balter, M. Chaplain, J. A. Glazier, and C. J. Weijer, Modeling Gastrulation in the Chick Embryo: Formation of the Primitive Streak, PLoS One 5, e10571 (2010).

[16] A. J. Ewald, A. Brenot, M. Duong, B. S. Chan, and Z. Werb, Collective Epithelial Migration and Cell Rearrangements
Drive Mammary Branching Morphogenesis, Dev. Cell 14, 570 (2008).

[17] A. Fatehullah, P. L. Appleton, and I. S. Näthke, Cell and Tissue Polarity in the Intestinal Tract During Tumourigenesis: Cells Still Know the Right Way up, But Tissue Organization Is Lost, Phil. Trans. R. Soc. B 368, 20130014 (2013).

[18] L. Ritsma, S. I. J. Ellenbroek, A. Zomer, H. J. Snippert, F. J. de Sauvage, B. D. Simons, H. Clevers, and J. van Rheenen, Intestinal Crypt Homeostasis Revealed at Single-Stem-Cell Level by In Vivo Live Imaging, Nature (London) 507, 362 (2014).

[19] J. M. Collinson, L. Morris, A. I. Reid, T. Ramaesh, M. A. Keighren, J. H. Flockhart, R. E. Hill, S.-S. Tan, K. Ramaesh, B. Dhillon, and J. D. West, Clonal Analysis of Patterns of Growth, Stem Cell Activity, and Cell Movement During the Development and Maintenance of the Murine Corneal Epithelium, Dev. Dyn. 224, 432 (2002).

[20] M. Mishra, Y. Huang, P. Srivastava, R. Srinivasan, M. Sevugan, R. Shlomovitz, N. Gov, M. Rao, and M. Balasubramanian, Cylindrical Cellular Geometry Ensures Fidelity of Division Site Placement in Fission Yeast, J. Cell Sci. 125, 3850 (2012).

[21] H. G. Yevick, G. Duclos, I. Bonnet, and P. Silberzan, Architecture and Migration of an Epithelium on a Cylindrical Wire, Proc. Natl. Acad. Sci. U.S.A. 112, 5944 (2015).

[22] Y. Fily, A. Baskaran, and M. F. Hagan, Dynamics of SelfPropelled Particles under Strong Confinement, Soft Matter 10, 5609 (2014).

[23] Y. Fily, A. Baskaran, and M. F. Hagan, Dynamics and Density Distribution of Strongly Confined Noninteracting Nonaligning Self-Propelled Particles in a Nonconvex Boundary, Phys. Rev. E 91, 012125 (2015).

[24] Y. Fily, A. Baskaran, and M. F. Hagan, Equilibrium Mappings in Polar-Isotropic Confined Active Particles, arXiv:1612.08719.

[25] N. Nikola, A. P. Solon, Y. Kafri, M. Kardar, J. Tailleur, and R. Voituriez, Active Particles with Soft and Curved Walls: Equation of State, Ratchets, and Instabilities, Phys. Rev. Lett. 117, 098001 (2016).

[26] F. Smallenburg and H. Löwen, Swim Pressure on Walls with Curves and Corners, Phys. Rev. E 92, 032304 (2015).

[27] R. Green, J. Toner, and V. Vitelli, The Geometry of Threshold-less Active Flow in Nematic Microfluidics, arXiv:1602.00561.

[28] Y. Fily, A. Baskaran, and M. F. Hagan, Active Particles on Curved Surfaces, arXiv:1601.00324.

[29] R. Sknepnek and S. Henkes, Active Swarms on a Sphere, Phys. Rev. E 91, 022306 (2015).

[30] D. R. Nelson, Defects and Geometry in Condensed Matter Physics (Cambridge University Press, Cambridge, England, 2002).

[31] A. Maitra, P. Srivastava, M. Rao, and S. Ramaswamy, Activating Membranes, Phys. Rev. Lett. 112, 258101 (2014).

[32] S. A. Mallory, C. Valeriani, and A. Cacciuto, CurvatureInduced Activation of a Passive Tracer in an Active Bath, Phys. Rev. E 90, 032309 (2014). 
[33] A. Souslov, B. C. van Zuiden, D. Bartolo, and V. Vitelli, Topological Sound in Active-Liquid Metamaterials, arXiv:1610.06873.

[34] Z. Yang, F. Gao, X. Shi, X. Lin, Z. Gao, Y. Chong, and B. Zhang, Topological Acoustics, Phys. Rev. Lett. 114, 114301 (2015).

[35] E. Prodan and C. Prodan, Topological Phonon Modes and Their Role in Dynamic Instability of Microtubules, Phys. Rev. Lett. 103, 248101 (2009).

[36] C. L. Kane and T. C. Lubensky, Topological Boundary Modes in Isostatic Lattices, Nat. Phys. 10, 39 (2014).

[37] S. Raghu and F. D. M. Haldane, Analogs of Quantum-HallEffect Edge States in Photonic Crystals, Phys. Rev. A 78, 033834 (2008).

[38] M.Z. Hasan and C. L. Kane, Colloquium: Topological Insulators, Rev. Mod. Phys. 82, 3045 (2010).

[39] P. Delplace, J. B. Marston, and A. Venaille, Topological Origin of Geophysical Waves, arXiv:1702.07583.

[40] P. Srivastava, R. Shlomovitz, N. S. Gov, and M. Rao, Patterning of Polar Active Filaments on a Tense Cylindrical Membrane, Phys. Rev. Lett. 110, 168104 (2013).

[41] M. Eisenberg and R. Guy, A Proof of the Hairy Ball Theorem, Am. Math. Mon. 86, 571 (1979).

[42] Y. Tu, J. Toner, and M. Ulm, Sound Waves and the Absence of Galilean Invariance in Flocks, Phys. Rev. Lett. 80, 4819 (1998).

[43] J. Toner and Y. Tu, Long-Range Order in a TwoDimensional Dynamical XY Model: How Birds Fly Together, Phys. Rev. Lett. 75, 4326 (1995).

[44] J. Toner and Y. Tu, Flocks, Herds, and Schools: A Quantitative Theory of Flocking, Phys. Rev. E 58, 4828 (1998).

[45] Manfredo Perdigao do Carmo Valero, Riemannian Geometry (Prentice-Hall, Englewood Cliffs, NJ, 1992).

[46] X. Yang, M. L. Manning, and M. C. Marchetti, Aggregation and Segregation of Confined Active Particles, Soft Matter 10, 6477 (2014).

[47] S. C. Takatori, W. Yan, and J. F. Brady, Swim Pressure: Stress Generation in Active Matter, Phys. Rev. Lett. 113, 028103 (2014).

[48] J. Toner, Reanalysis of the Hydrodynamic Theory of Fluid and Polar-Ordered Flocks, Phys. Rev. E 86, 031918 (2012).

[49] A. Gopinath, M. F. Hagan, M. C. Marchetti, and A. Baskaran, Dynamical Self-Regulation in Self-Propelled Particle Flows, Phys. Rev. E 85, 061903 (2012).

[50] E. Bertin, M. Droz, and G. Grégoire, Hydrodynamic Equations for Self-Propelled Particles: Microscopic Derivation and Stability Analysis, J. Phys. A 42, 445001 (2009).

[51] A. P. Solon, J.-B. Caussin, D. Bartolo, H. Chaté, and J. Tailleur, Pattern Formation in Flocking Models: A Hydrodynamic Description, Phys. Rev. E 92, 062111 (2015).

[52] J.-B. Caussin, A. Solon, A. Peshkov, H. Chaté, T. Dauxois, J. Tailleur, V. Vitelli, and D. Bartolo, Emergent Spatial Structures in Flocking Models: A Dynamical System Insight, Phys. Rev. Lett. 112, 148102 (2014).

[53] S. Mishra, A. Baskaran, and M. C. Marchetti, Fluctuations and Pattern Formation in Self-Propelled Particles, Phys. Rev. E 81, 061916 (2010).
[54] E. Bertin, M. Droz, and G. Grégoire, Boltzmann and Hydrodynamic Description for Self-Propelled Particles, Phys. Rev. E 74, 022101 (2006).

[55] P. Gilles De Gennes and J. Prost, The Physics of Liquid Crystals (Clarendon Press, Oxford, 1993).

[56] W. Kung, M. C. Marchetti, and K. Saunders, Hydrodynamics of Polar Liquid Crystals, Phys. Rev. E 73, 031708 (2006).

[57] A. Baskaran and M. C. Marchetti, Hydrodynamics of SelfPropelled Hard Rods, Phys. Rev. E 77, 011920 (2008).

[58] P. C. Martin, O. Parodi, and P. S. Pershan, Unified Hydrodynamic Theory for Crystals, Liquid Crystals, and Normal Fluids, Phys. Rev. A 6, 2401 (1972).

[59] A.E. Gill, Atmosphere-Ocean Dynamics (Elsevier, New York, 2016).

[60] The term involving $\bar{\lambda}(\eta+2) m \mathrm{v}$ is not relevant as its contribution to the dispersion is subdominant, as can be seen from its absence in Eq. (25).

[61] M. V. Berry, Quantal Phase Factors Accompanying Adiabatic Changes, Proc. R. Soc. A 392, 45 (1984).

[62] M. Nakahara, Geometry, Topology and Physics (Taylor \& Francis, London, 2003).

[63] F. D. M. Haldane, Model for a Quantum Hall Effect without Landau Levels: Condensed-Matter Realization of the "Parity Anomaly”, Phys. Rev. Lett. 61, 2015 (1988).

[64] S. Ryu, A. P. Schnyder, A. Furusaki, and A. W. W. Ludwig, Topological Insulators and Superconductors: Tenfold Way and Dimensional Hierarchy, New J. Phys. 12, 065010 (2010).

[65] D. Leykam, K. Y. Bliokh, C. Huang, Y. D. Chong, and F. Nori, Edge Modes, Degeneracies, and Topological Numbers in Non-Hermitian Systems, Phys. Rev. Lett. 118, 040401 (2017).

[66] R. Jackiw and C. Rebbi, Solitons with Fermion Number 1/2, Phys. Rev. D 13, 3398 (1976).

[67] More precisely, one needs a Killing field on the surface dictating a symmetry direction. One can always have the Christoffel symbols vanish at a given point in Riemann normal coordinates, but in the presence of a Killing field, this extends to an integral curve along which the Christoffel symbols vanish, changing sign as it is crossed. This provides the necessary structure for the existence of such topological modes.

[68] M. J. Bowick and L. Giomi, Two-Dimensional Matter: Order, Curvature and Defects, Adv. Phys. 58, 449 (2009).

[69] A. M. Turner, V. Vitelli, and D. R. Nelson, Vortices on Curved Surfaces, Rev. Mod. Phys. 82, 1301 (2010).

[70] M. J. Bowick, D. R. Nelson, and A. Travesset, Interacting Topological Defects on Frozen Topographies, Phys. Rev. B 62, 8738 (2000).

[71] A. R. Bausch, M. J. Bowick, A. Cacciuto, A. D. Dinsmore, M. F. Hsu, D. R. Nelson, M. G. Nikolaides, A. Travesset, and D. A. Weitz, Grain Boundary Scars and Spherical Crystallography, Science 299, 1716 (2003).

[72] W. T. M. Irvine, V. Vitelli, and P. M. Chaikin, Pleats in Crystals on Curved Surfaces, Nature (London) 468, 947 (2010).

[73] M. J. Bowick and Z. Yao, Crystalline Order on Catenoidal Capillary Bridges, Europhys. Lett. 93, 36001 (2011). 
[74] E. Bendito, M. J. Bowick, A. Medina, and Z. Yao, Crystalline Particle Packings on Constant Mean Curvature (Delaunay) Surfaces, Phys. Rev. E 88, 012405 (2013).

[75] D. R. Nelson and L. Peliti, Fluctuations in Membranes with Crystalline and Hexatic Order, J. Phys. II (France) 48, 1085 (1987).

[76] H. S. Seung and D. R. Nelson, Defects in Flexible Membranes with Crystalline Order, Phys. Rev. A 38, 1005 (1988).

[77] X. Xing, H. Shin, M. J. Bowick, Z. Yao, L. Jia, and M.-H. Li, Morphology of Nematic and Smectic Vesicles, Proc. Natl. Acad. Sci. U.S.A. 109, 5202 (2012).

[78] N. Ramakrishnan, J. H. Ipsen, and P. B. Sunil Kumar, Role of Disclinations in Determining the Morphology of Deformable Fluid Interfaces, Soft Matter 8, 3058 (2012).

[79] L. Mesarec, P. Kurioz, and A. Iglič, W. Góźdź, and S. Kralj, Curvature-Controlled Topological Defects, Crystals 7, 153 (2017).

[80] D. R. Nelson, Liquids and Glasses in Spaces of Incommensurate Curvature, Phys. Rev. Lett. 50, 982 (1983).
[81] C. D. Modes and R. D. Kamien, Hard Disks on the Hyperbolic Plane, Phys. Rev. Lett. 99, 235701 (2007).

[82] V. Vitelli and A. M. Turner, Anomalous Coupling Between Topological Defects and Curvature, Phys. Rev. Lett. 93, 215301 (2004).

[83] L. M. Nash, D. Kleckner, A. Read, V. Vitelli, A. M. Turner, and W. T. M. Irvine, Topological Mechanics of Gyroscopic Metamaterials, Proc. Natl. Acad. Sci. U.S.A. 112, 14495 (2015).

[84] X. Serra-Picamal, V. Conte, R. Vincent, E. Anon, D. T. Tambe, E. Bazellieres, J. P. Butler, J. J. Fredberg, and X. Trepat, Mechanical Waves During Tissue Expansion, Nat. Phys. 8, 628 (2012).

[85] F. E. O. Holmberg, J. B. Seidelin, X. Yin, B. E. Mead, Z. Tong, Y. Li, J. M. Karp, and O. H. Nielsen, Culturing Human Intestinal Stem Cells for Regenerative Applications in the Treatment of Inflammatory Bowel Disease, EMBO Mol. Med. 9, 558 (2017).

[86] S. Hopyan, J. Sharpe, and Y.Yang, Budding Behaviors: Growth of the Limb as a Model of Morphogenesis, Dev. Dyn. 240, 1054 (2011). 